

\title{
IMPLANTAÇÃO DA ABNT NBR ISO/IEC 17025: UMA ABORDAGEM POR GERENCIAMENTO DE PROJETOS
}

\author{
IMPLEMENTATION OF ABNT NBR ISO/IEC 17025: A PROJECT MANAGEMENT \\ APPROACH
}

\author{
iD Pedro Henrique Tascheto Santos ${ }^{1}$ \\ iD Morgana Pizzolato ${ }^{2}$ \\ Marlon Soliman ${ }^{3}$
}

${ }^{1}$ Graduando em Engenharia de Produção Universidade Federal de Santa Maria - UFSM Santa Maria, RS - Brasil. pedrohtsantos@gmail.com

2 Doutora em Engenharia de Produção Universidade Federal de Santa Maria - UFSM Santa Maria, RS - Brasil. morganapizzolato@ufsm.br

3 Doutor em Engenharia de Produção Universidade Federal de Santa Maria - UFSM Santa Maria, RS - Brasil. marlon.soliman@ufsm.br

Recebido em: 26 jul. 2020

Aprovado em: 25 out. 2020
Resumo: Este estudo tem como objetivo estruturar a implantação de um Sistemas de Gestão da Qualidade (SGQ) baseado na norma ISO/IEC 17025 em laboratórios de ensaio e calibração de uma instituição de ensino superior (IES) utilizando a abordagem de gerenciamento de projetos (GP). O método de pesquisa foi composto de: (i) definição da abordagem de GP a ser utilizada; (ii) definição dos pacotes de trabalho; (iii) construção da estrutura analítica do projeto; (iv) identificação das relações de dependência; e (v) identificação de práticas e ferramentas de GP aplicáveis. Os resultados apresentam uma estrutura que esclarece o trabalho a ser feito para a implantação de SGQs baseados na norma ISO/IEC 17025, contribuindo para a teoria ao demonstrar como as metodologias de GP se aplicam nesse contexto. Para a prática, gestores e decisores podem se beneficiar utilizando o planejamento apresentado como base para seus projetos de implantação desta e de outras normas. Para a sociedade, espera-se que os resultados beneficiem especialmente laboratórios de IES, os quais atendem tanto a comunidade acadêmica quanto a comunidade em geral.

Palavras-chave: Gerenciamento de projetos. Sistemas de gestão da qualidade. ABNT NBR ISO/IEC 17025. Laboratórios.

Abstract: This study aims to structure the implementation of a Quality Management Systems (QMS) based on the ISO/IEC 17025 standard in testing and calibration laboratories of a higher education institution (HEI) using the project management (PM) approach. The research method was composed of five stages: (i) definition of the PM approach to be used; (ii) definition of work packages; (iii) construction of the project's analytical structure (WBS); (iv) identification of dependency relationships; and (v) identification of PM practices and tools applicable. The results present a structure that clarifies the work to be done for the implementation of QMS based on the ISO/IEC 17025 standard, contributing to the theory by demonstrating how GP methodologies apply in this context. For practice, managers and decision makers can benefit from using the planning presented as the basis for their projects to implement this and other standards. For society, the results are expected to benefit especially HEI laboratories, which serve both the academic community and the community in general.

Keywords: Project management. Quality management systems. ISO/IEC 17025. Laboratories. 
1 Introdução

Resultados de ensaios e calibrações realizados por laboratórios estão frequentemente presentes no cotidiano das pessoas, seja por meio da medição correta da quantidade de energia consumida em uma residência ou até mesmo na qualidade da água distribuída pela rede pública de uma cidade. Visando a confiança na operação de laboratórios, a norma ABNT NBR ISO/IEC 17025 propõe a implantação de Sistemas de Gestão da Qualidade (SGQ), os quais permitem que os laboratórios demonstrem sua competência e a validade dos resultados (ABNT, 2017).

Segundo Grochau e Caten (2012), ainda que exista um grande auxílio para a implantação de SGQ por meio de órgãos acreditadores, empresas privadas e outros institutos, muitos laboratórios acabam por contratar serviços externos para guiá-los neste processo. Esta dificuldade fica ainda mais evidente, por exemplo, quando se trata de laboratórios de Instituições de Ensino Superior (IES), onde o foco principal nem sempre é a prestação de serviços, visto que muitos deles também estão envolvidos em atividades de ensino e pesquisa (Grochau et al., 2010; Lovrenčić Mikelić, 2020; Rodima et al., 2005; Zapata-García et al., 2007). Como consequência dessa dificuldade, em um levantamento realizado em 2015, foi constatado que dos 1.009 laboratórios de ensaio e calibração acreditados pela Coordenação Geral de Acreditação do INMETRO (CGCRE), organismo responsável pela acreditação de laboratórios no Brasil, apenas $11 \%$ pertenciam às IES (Grochau et al., 2015).

A implantação de um sistema de gestão da qualidade baseado na norma ISSO/IEC 17025 em laboratórios de IES é vista como uma oportunidade de geração de receita, contribuindo para sua sustentabilidade, embora o processo de implantação ainda seja um desafio nesse contexto (Aqidawati et al., 2019). Com o intuito de estruturar e guiar a implantação de Sistemas de Gestão da Qualidade baseados na norma ABNT NBR ISO/IEC 17025, autores como Grochau e Caten (2012) e Rauret e Compañó (2003), sugerem que a implantação de SGQ possa ser realizada por meio de uma abordagem de processo. Entretanto, também é possível abordar a implantação e manutenção de SGQ como um projeto, uma vez que, por definição, um projeto é considerado "um esforço temporário empreendido para criar um produto, serviço ou resultado exclusivo"(PMI, 2018). A literatura referente à implantação de sistemas de gestão da qualidade, no entanto, ainda é bastante escassa quanto a utilização do corpo de conhecimentos da gestão de projetos para esse fim (Ingason, 2015). No caso da implantação da ABNT NBR ISO/IEC 17025, tal projeto, ao seu final, resulta em um SGQ operante em laboratórios de calibração e ensaios.

Assim, essa pesquisa originou-se da seguinte questão: como aprimorar o processo de implantação Sistemas de Gestão da Qualidade baseados na norma ABNT NBR ISO/IEC 17025 em laboratórios de ensaio e calibração? Para responder à pergunta, essa pesquisa teve como objetivo 
estruturar a implantação de um SGQ baseados na norma ABNT NBR ISO/IEC 17025 em laboratórios de ensaio e calibração de uma Instituição de Ensino Superior utilizando a abordagem de gestão de projetos.

É possível justificar o desenvolvimento desta pesquisa em dois contextos, teórico e prático. Para o primeiro, diversos autores tratam em seus estudos da implantação de normas e SGQ (Chung et al., 2006; Grochau \& ten Caten, 2012; Lopes et al., 2014; Netto et al., 2009; Rauret \& Compañó, 2003; Salgueiro, 2012; Zapata-García et al., 2007). Entretanto, dentre os autores que desenvolveram seus estudos em IES, nenhum aborda uma perspectiva de gerenciamento de projetos para esta implantação. Por mais que Rauret e Compañó (2003) nomeiem a implantação da norma ISO/IEC 17025 como um projeto, eles acabam utilizando uma abordagem de processo para fazê-la.

Para o contexto prático, o estudo se justifica quanto à importância que a implantação do SGQ e, por conseguinte, da norma ABNT NBR ISO/IEC 17025 tem para laboratórios e para a sociedade acadêmica (Aqidawati et al., 2019). Profissionais envolvidos na implantação de sistemas de gestão da qualidade podem se beneficiar dos resultados dessa pesquisa, que demonstram como a norma ISO/IEC 17025 pode ser desmembrada em pacotes de trabalho, deixando claro o trabalho a ser feito na sua implantação. Ainda, os resultados apresentados trazem uma estrutura analítica de projeto e as relações de dependência entre os diversos itens que compõem essa estrutura. Com isso, gestores e decisores que estejam a frente da implantação de SGQs baseados na norma ISO/IEC 17025 podem também fazer uso dos resultados dessa pesquisa em diferentes contextos, tomando como base o planejamento apresentado nessa pesquisa para proceder com a execução, respeitando as particularidades de cada caso, fazendo uso eficiente dos recursos disponíveis e aumentando as chances de sucesso.

\section{Norma ABNT NBR ISO/IEC 17025}

A norma ABNT NBR ISO/IEC 17025 surgiu a partir da necessidade de se amparar os resultados de laboratórios de ensaio e/ou calibração e promover a confiança em suas operações. Atualmente, a norma está em sua terceira edição e encontra-se alinhada aos princípios da ABNT NBR ISO 9001:2015. Assim, pode-se observar que a norma abrange tanto as competências técnicas necessárias aos laboratórios, quanto os requisitos do sistema de gestão da qualidade (ABNT, 2017; Albano \& RayaRodriguez, 2009; Felippes et al., 2011).

Aplicável a organizações de qualquer tamanho que efetuem atividades de laboratório, o documento estabelece requisitos em cinco categorias: requisitos gerais; requisitos de estrutura; requisitos de recursos; requisitos de processo; e, requisitos do sistema de gestão (ABNT, 2017). Segundo o INMETRO (2018), ainda é possível classificar os requisitos apresentados entre aspectos técnicos, os quais evidenciam a capacidade técnica para realização das atividades de laboratório, e aspectos da gerência, os quais evidenciam a capacidade do SGQ. 
Grochau e ten Caten (2012) sugerem que a implantação de SGQ baseados na ISO/IEC 17025 siga nove passos: (i) pré-definir equipe e escopo da acreditação; (ii) realizar diagnóstico da situação atual; (iii) calcular os custos da implantação; (iv) elaborar cronograma e definir responsabilidades; (v) mapear e analisar processos; (vi) estabelecer os requisitos de gerenciamento do SGQ; (vii) estabelecer os requisitos técnicos do SGQ; (viii) definir e acompanhar indicadores; e (ix) avaliar o SGQ. A avaliação do SGQ implantado deve ser realizada por pessoal capacitado, e deve cobrir todos os aspectos do SGQ. Com o sistema auditado e as melhorias implantadas, pode-se afirmar que o sistema está apto a começar seu processo de acreditação (Grochau e ten Caten, 2012).

\section{Gerenciamento de projetos}

O Gerenciamento de Projetos (GP) tem se destacado como uma das principais estratégias de gestão do mundo moderno (Kerzner, 2015; Vargas, 2018), sendo inclusive indicado como uma forma de superar lacunas culturais quando da implantação de novas práticas (Barnes et al., 2006; Fernandes et al., 2020). Segundo o PMI (2018), GP é o uso de um conjunto de ferramentas, habilidades e conhecimentos durante as atividades de um projeto. Para Vargas (2018), a sua função consiste no estabelecimento de um processo estruturado e lógico, capaz de administrar eventos com alto nível de novidade, complexidade e dinamismo. Devido à grande adaptabilidade do gerenciamento de projetos, é comum que cada organização ou empresa adote uma metodologia particular para sua realidade. Entretanto, é crucial que exista um alinhamento entre atividades de GP e as estratégias e objetivos da organização para o sucesso de seus projetos (Vargas, 2018).

Ao longo dos anos, diversas associações de profissionais da área foram estabelecidas, tais como o PMI , nos Estados Unidos, o Australian Institute for Project Management (AIPM), na Austrália, e a Association for Project Management (APM), no Reino Unido. Estas organizações foram responsáveis por padronizar, agregar e disseminar os fundamentos de GP nos chamados BOKs, sigla em inglês para "Bodies of Knowledges" e, dentre eles, o Guia do Conhecimento em Gerenciamento de Projetos (Guia $\mathrm{PMBOK}^{\circ}$ ), elaborado pelo $\mathrm{PMI}^{\circ}$, é o mais difundido (Eder et al., 2012). Atualmente, estes guias são comumente classificados como abordagem tradicional de GP. A abordagem tradicional, também chamada de heavyweight, apresenta como principais características estruturas rígidas de escopo, prazos e custos. O enfoque dessa abordagem concentra-se no planejamento, gerando um alto volume de documentação. Por essas razões, a abordagem tradicional é considerada rígida e burocrática, sendo muitas vezes inadequada para ambientes dinâmicos e incertos (Menezes, 2018).

No contraponto à abordagem tradicional, surge a partir dos anos 1990 a abordagem ágil, também conhecida como lightwheight. Segundo Highsmith (2009), o Gerenciamento Ágil de Projetos (GAP) pode ser definido como "um conjunto de princípios, valores e práticas que auxiliam a equipe de 
projetos a entregar produtos ou serviços de valor em um ambiente de projetos desafiador". A abordagem ágil preconiza o trabalho sem estruturas rígidas, com foco na execução e fluxo contínuo de desenvolvimento (Eder et al., 2014). No entanto, sua aplicação desestruturada pode ser incompatível para projetos que precisem comprovar a rastreabilidade das decisões tomadas e prestações de contas, uma vez que a documentação gerada é mínima (Menezes, 2018).

Pela alta complexidade envolvida em ambientes de projetos, é difícil que um modelo padrão ou até mesmo um conjunto de boas práticas se aplique a qualquer realidade. Assim, muitas organizações optam por uma abordagem híbrida de GP, que utiliza um misto de práticas tradicionais e ágeis visando um resultado mais eficiente (Cruz, 2013; Jackson, 2012). É importante ressaltar que para atingir resultados positivos por meio de uma abordagem híbrida, é necessário escolher uma abordagem dominante à qual a segunda será adicionada (Cruz, 2013).

\section{Metodologia}

A aplicação da presente pesquisa foi desenvolvida no Sistema de Gestão dos Laboratórios do Centro de Tecnologia (SGLab CT) da Universidade Federal de Santa Maria (UFSM). O SGLab CT é um núcleo ligado à Direção do Centro de Tecnologia (CT) e compreende diversos laboratórios técnicocientíficos de diferentes áreas da engenharia e tecnologias (SGLab, 2020). Desenvolvido por professores, técnicos e estudantes de graduação e pós-graduação da instituição, o sistema de gestão da qualidade do SGLab CT tem como base a ABNT NBR ISO/IEC 17025.

A estrutura do SGLab CT é composta por três grupos: a Gerência, o Escritório da Qualidade, e os laboratórios. O primeiro é composto pelo Diretor do Centro de Tecnologia e pelos Coordenadores e/ou Diretores dos laboratórios. O Escritório da Qualidade (EQ) é liderado pelo Gerente da Qualidade (GQ), o qual conta com o apoio de uma equipe de analistas da qualidade e assistentes administrativos. Por fim, os laboratórios associados ao SGLab CT têm em suas equipes um Gerente Técnico (GT), o qual é responsável por coordenar as operações técnicas do laboratório, e podem optar por ter um Gerente Administrativo (GA), o qual é encarregado de coordenar as operações administrativas do laboratório.

A realização desse estudo está estruturada em cinco etapas, as quais são: (i) definição da abordagem de GP a ser utilizada; (ii) definição dos pacotes de trabalho; (iii) construção da estrutura analítica do projeto (EAP); (iv) identificação das relações de dependência; e (v) identificação de práticas e ferramentas de GP aplicáveis à implantação de um SGQ baseado na ABNT NBR ISO/IEC 17025. Para tanto, na etapa (i) buscou-se definir qual abordagem de GP (tradicional, ágil ou híbrida) seria a mais indicada para o caso. Essa etapa foi realizada por meio de reuniões juntamente da equipe do Escritório da Qualidade, analisando-se as características de cada abordagem e como estas influenciariam o andamento das atividades dentro do SGLab CT. Para a etapa (ii), realizou-se uma extensa análise da 
norma, buscando compreender como seus requisitos se inter-relacionam e quais as ações necessárias para atendê-los. A partir disso, um mapa mental da interrelação entre os requisitos foi desenvolvido, o que permitiu a identificação de pacotes de trabalho para cumprir com os mesmos.

Para a etapa (iii), os pacotes de trabalho foram classificados hierarquicamente, formando-se a estrutura analítica do projeto (EAP). Embora existam diferentes formas de construção de uma EAP, adotou-se nesse estudo a EAP baseada no ciclo de vida, onde as fases do projeto formam o nível 2 da estrutura. Esse tipo de EAP permite uma visualização clara do desenvolvimento do projeto ao longo do tempo. Já para a etapa (iv), buscou-se estabelecer as relações de dependência entre os pacotes de trabalho. Essa etapa tem por objetivo esclarecer quais pacotes de trabalho possuem antecessoras e sucessoras, de modo que a implantação da norma se torne inequívoca quanto a precedência dos pacotes de trabalho e seu sequenciamento. Por fim, a etapa (v) buscou evidenciar possíveis práticas e ferramentas de GP que podem ser úteis em cada fase do projeto, de modo que a implantação da norma possa ocorrer com eficiência e eficácia.

\section{Resultados}

\subsection{Definição da abordagem de GP a ser utilizada}

Relativo a abordagens tradicionais, elencou-se que suas principais vantagens seriam: foco no escopo do projeto e na entrega total, visto que o projeto envolve a implantação de uma norma onde todos seus requisitos devem ser efetivamente atendidos; e, maior enfoque na etapa de planejamento, uma vez que ele colabora para a diminuição de riscos e possíveis não conformidades do SGQ em relação aos requisitos da norma. Como desvantagens de abordagens tradicionais, identificou-se: alta centralização dos processos no gerente do projeto, em virtude de muitos colaboradores acumularem funções dentro do SGLab CT e/ou universidade; e, complexidade e abrangência, dado o elevado número de processos envolvidos, dos quais nem todos são aplicáveis a realidade do SGLab CT.

Quanto a abordagens ágeis, foram identificadas como principais vantagens: uso de ferramentas visuais para acompanhamento e definição do projeto, posto que diversos colaboradores do SGLab CT relatam dificuldade no entendimento do processo de implantação do SGQ; e, auto-gestão, autoorganização e comunicação entre os membros da equipe do projeto, em virtude de facilitar o desenvolvimento das atividades. Referente às desvantagens desta abordagem, destacou-se: planejamento simplório, uma vez que um planejamento ineficiente pode gerar não conformidades do sistema em relação aos requisitos da norma; divisão do backlog do projeto em backlog dos sprints, devido ao fato de muitas das atividades de implantação do SGQ estarem diretamente relacionadas.

No tocante a abordagens híbridas, reconheceu-se como principal vantagem a possibilidade de adaptar e unir os pontos fortes das abordagens anteriores, uma vez que ambas apresentam práticas 
complementares para o atendimento às peculiaridades do projeto. Com base na análise dos pontos destacados, optou-se pela utilização de uma metodologia híbrida com a abordagem tradicional como dominante, onde essa seria baseada no Guia PMBOK ${ }^{\circ}$. Esta escolha baseou-se na necessidade do projeto ser focado em seu escopo e sua entrega total, visto que todas as etapas devem ser entregues para real implantação da norma.

\subsection{Definição dos pacotes de trabalho}

A partir da análise da norma, da interrelação entre seus requisitos e das ações necessárias para atendê-los, classificou-se o tipo destas relações como direta ou indireta. Requisitos com relação direta são aqueles que têm ações para implantação fundamentalmente associadas, as quais podem, na maioria dos casos, ser unificadas. Já requisitos com relação indireta não necessariamente implicam em ações para a implantação da norma, estes geralmente envolvem diretrizes para o dia-a-dia do laboratório. Além disso, estes requisitos foram agrupados em áreas de afinidade, as quais são relacionadas aos processos do SGQ, e cada área pode conter requisitos de uma ou mais subseções da norma.

A partir desta análise, elaborou-se um mapa mental destes requisitos, o qual pode ser visualizado na Figura 1. 
Figura 1

Mapa mental dos requisitos da ISO/IEC 170

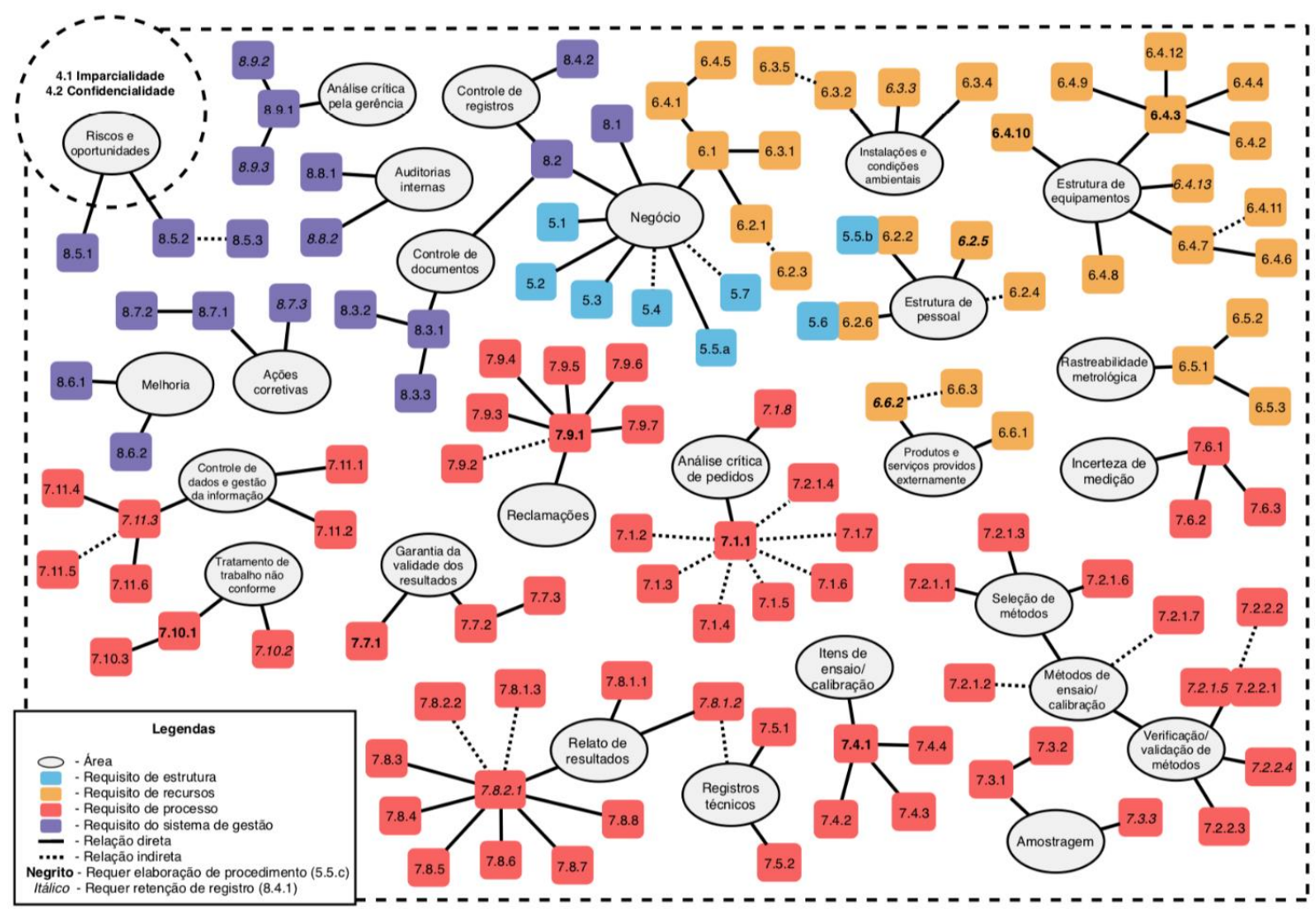

Fonte: Elaborada pelos autores

Ressalta-se que, os requisitos "4.1 Imparcialidade", "4.2 Confidencialidade" e a área de afinidade "Riscos e oportunidades" estão indiretamente relacionados a todos os requisitos da norma. Além disso, todos os requisitos que estabelecem a elaboração de procedimentos e os que requerem a retenção de registros estão indiretamente relacionados aos requisitos 5.5.c e 8.4.1, respectivamente. Com base no mapa mental elaborado, estabeleceu-se 58 pacotes de trabalho relacionados à implantação da norma. Distribuídos entre 23 áreas do SGQ, os pacotes de trabalho são apresentados na Tabela 1. 


\section{Tabela 1}

Pacotes de trabalho para implantação da ISO/IEC 17025

\begin{tabular}{|c|c|c|}
\hline Área de afinidade & Pacote de trabalho & $\begin{array}{l}\text { Requisito(s) } \\
\text { relacionado(s) }\end{array}$ \\
\hline \multirow{8}{*}{ Negócio } & Estabelecer laboratório & 5.1 \\
\hline & Definir estrutura organizacional & 5.5.a \\
\hline & Selecionar pessoal (Inicial) & $6.1 ; 6.2 .1 ; 6.2 .3$ \\
\hline & Definir gerência & 5.2 \\
\hline & Definir escopo de acreditação & 5.3 \\
\hline & Aquisição de equipamentos & $6.1 ; 6.4 .1 ; 6.4 .5$ \\
\hline & Instalações físicas & 6.3 .1 \\
\hline & Diretrizes do sistema & $4.1 ; 4.2 ; 5.4 ; 5.7 ; 8.1 ; 8.2$ \\
\hline \multirow{2}{*}{$\begin{array}{l}\text { Documentos e } \\
\text { registros do sistema }\end{array}$} & Sistemática de documentos & 8.3.1; 8.3.2 \\
\hline & Sistemática de registros & $8.4 .1 ; 8.4 .2$ \\
\hline \multirow{4}{*}{ Pessoal } & Definir procedimento(s) de pessoal & 5.5.c; 6.2 .5 \\
\hline & $\begin{array}{l}\text { Requisitos de competência, } \\
\text { responsabilidades, autoridades e inter- } \\
\text { relacionamento }\end{array}$ & 5.5.b; 6.2.2; 6.2.4 \\
\hline & Estabelecer registros de pessoal & $6.2 .5 ; 8.4 .1$ \\
\hline & Autorizar pessoal & $5.6 ; 6.2 .6$ \\
\hline \multirow{3}{*}{$\begin{array}{l}\text { Instalações e } \\
\text { condições ambientais }\end{array}$} & $\begin{array}{l}\text { Documentar requisitos para instalações e } \\
\text { condições ambientais }\end{array}$ & $6.3 .2 ; 6.3 .5$ \\
\hline & Medidas de controle das instalações & 6.3 .4 \\
\hline & $\begin{array}{l}\text { Monitoramento, controle e registro das } \\
\text { condições ambientais }\end{array}$ & $6.3 .3 ; 8.4 .1$ \\
\hline \multirow{5}{*}{ Equipamentos } & Procedimento de equipamentos & $\begin{array}{l}\text { 5.5.c; } 6.4 .2 ; 6.4 .3 ; 6.4 .4 \\
6.4 .9 ; 6.4 .12\end{array}$ \\
\hline & Identificação de equipamentos & 6.4 .8 \\
\hline & Estabelecer registros de equipamentos & $6.4 .12 ; 8.4 .1$ \\
\hline & Programa de calibração & $6.4 .6 ; 6.4 .7$ \\
\hline & $\begin{array}{l}\text { Definir procedimento para checagens } \\
\text { intermediárias }\end{array}$ & 5.5.c; 6.4 .10 \\
\hline $\begin{array}{l}\text { Rastreabilidade } \\
\text { metrológica }\end{array}$ & Rastreabilidade metrológica & $6.5 .1 ; 6.5 .2 ; 6.5 .3$ \\
\hline \multirow{3}{*}{$\begin{array}{l}\text { Produtos e serviços } \\
\text { providos } \\
\text { externamente }\end{array}$} & $\begin{array}{l}\text { Definir procedimento de produtos e serviços } \\
\text { providos externamente }\end{array}$ & 5.5.c; $6.6 .2 ; 6.6 .3$ \\
\hline & $\begin{array}{l}\text { Estabelecer registros de produtos e serviços } \\
\text { providos externamente }\end{array}$ & $6.6 .2 ; 8.4 .1$ \\
\hline & Selecionar provedores externos & 6.6 .1 \\
\hline \multirow{4}{*}{ Métodos } & Seleção de métodos & 7.2 .1 \\
\hline & $\begin{array}{l}\text { Estabelecer sistemática de } \\
\text { verificação/validação de métodos }\end{array}$ & 7.2.1.5; 7.2.2; 8.4.1 \\
\hline & Verificação e/ou validação de métodos & $\begin{array}{l}\text { 7.2.1.5; 7.2.2.1; 7.2.2.3; } \\
\text { 7.2.2.4 }\end{array}$ \\
\hline & Documentar métodos selecionados & 5.5.c; 7.2.1.2; 7.2.1.7 \\
\hline \multirow{2}{*}{ Amostragem } & Plano e método de amostragem & $7.3 .1 ; 7.3 .2$ \\
\hline & Estabelecer registros de amostragem & 7.3.3; 8.4.1 \\
\hline Incerteza de medição & Incerteza de medição & 7.6 \\
\hline
\end{tabular}




\begin{tabular}{|c|c|c|}
\hline Área de afinidade & Pacote de trabalho & $\begin{array}{l}\text { Requisito(s) } \\
\text { relacionado(s) }\end{array}$ \\
\hline \multirow{2}{*}{$\begin{array}{l}\text { Análise crítica de } \\
\text { pedidos, propostas e } \\
\text { contratos }\end{array}$} & $\begin{array}{l}\text { Definir procedimento de análise crítica de } \\
\text { pedidos, propostas e contratos }\end{array}$ & $\begin{array}{l}\text { 5.5.c; 7.1.1; 7.1.2; 7.1.3; } \\
\text { 7.1.4; 7.1.5; 7.1.6; 7.1.7; } \\
\text { 7.2.1.4 }\end{array}$ \\
\hline & Estabelecer registros de análises críticas & $7.1 .8 ; 8.4 .1$ \\
\hline $\begin{array}{l}\text { Manuseio de itens de } \\
\text { ensaio ou calibração }\end{array}$ & $\begin{array}{l}\text { Definir procedimento para itens de } \\
\text { ensaio/calibração }\end{array}$ & 5.5.c; 7.4 \\
\hline Registros técnicos & Registros técnicos & $7.5 .1 ; 7.5 .2$ \\
\hline \multirow{2}{*}{$\begin{array}{l}\text { Garantia da validade } \\
\text { dos resultados }\end{array}$} & $\begin{array}{l}\text { Definir procedimento para monitorar a } \\
\text { validade dos resultados }\end{array}$ & 5.5.c; 7.7.1 \\
\hline & Monitoramento do desempenho & 7.7.2; 7.7.3 \\
\hline \multirow{3}{*}{ Relato de resultados } & Sistemática para relato de resultados & $\begin{array}{l}\text { 7.8.1; 7.8.2; 7.8.3; 7.8.5; } \\
\text { 7.8.6; 7.8.7; 7.8.8 }\end{array}$ \\
\hline & Relatório de ensaio & $\begin{array}{l}7.5 ; 7.8 .1 ; 7.8 .2 ; 7.8 .3 \\
8.4 .1\end{array}$ \\
\hline & Certificado de calibração & $\begin{array}{l}7.5 ; 7.8 .1 ; 7.8 .2 ; 7.8 .4 \\
8.4 .1\end{array}$ \\
\hline Reclamações & Definir procedimento de reclamações & 5.5.c; 7.9 \\
\hline \multirow{2}{*}{$\begin{array}{l}\text { Trabalho não } \\
\text { conforme }\end{array}$} & $\begin{array}{l}\text { Definir procedimento de tratamento de } \\
\text { trabalho não conforme }\end{array}$ & 5.5.c; 7.10.1 \\
\hline & $\begin{array}{l}\text { Estabelecer registro de trabalho não } \\
\text { conforme }\end{array}$ & $7.10 .2 ; 8.4 .1$ \\
\hline \multirow{3}{*}{$\begin{array}{l}\text { Controle de dados e } \\
\text { gestão da informação }\end{array}$} & $\begin{array}{l}\text { Definir a sistemática de gestão da informação } \\
\text { laboratorial }\end{array}$ & 7.11 \\
\hline & $\begin{array}{l}\text { Estabelecer registros de sistema de gestão da } \\
\text { informação }\end{array}$ & 7.11.3.e; 8.4.1 \\
\hline & $\begin{array}{l}\text { Validar o sistema de gestão da informação e } \\
\text { controles de dados }\end{array}$ & 7.11 .2 \\
\hline \multirow{2}{*}{$\begin{array}{l}\text { Riscos e } \\
\text { oportunidades }\end{array}$} & $\begin{array}{l}\text { Identificar riscos e oportunidades associados } \\
\text { as atividades de laboratório }\end{array}$ & 8.5 .1 \\
\hline & $\begin{array}{l}\text { Planejar ações para abordar riscos e } \\
\text { oportunidades }\end{array}$ & $8.5 .2 ; 8.5 .3$ \\
\hline Melhoria & Melhoria & $8.6 .1 ; 8.6 .2$ \\
\hline \multirow[t]{2}{*}{ Ações corretivas } & $\begin{array}{l}\text { Estabelecer sistemática para tratamento de } \\
\text { não conformidades }\end{array}$ & 8.7.1; 8.7.2 \\
\hline & Estabelecer registros de ações corretivas & 8.4.1; 8.7.3 \\
\hline \multirow{2}{*}{ Auditoria internas } & Intervalo de auditorias internas & 8.8 .1 \\
\hline & Programa de auditoria interna & 8.8 .2 \\
\hline \multirow{3}{*}{$\begin{array}{l}\text { Análise crítica pela } \\
\text { gerência }\end{array}$} & Intervalo de análise crítica pela gerência & 8.9 .1 \\
\hline & $\begin{array}{l}\text { Estabelecer registro de entradas para análise } \\
\text { crítica pela gerência }\end{array}$ & $8.4 .1 ; 8.9 .2$ \\
\hline & $\begin{array}{l}\text { Estabelecer registro de saídas da análise } \\
\text { crítica pela gerência }\end{array}$ & $8.4 .1 ; 8.9 .3$ \\
\hline
\end{tabular}

Fonte: Elaborada pelos autores.

\subsection{Construção da estrutura analítica do projeto (EAP)}

Uma vez estabelecidas as ações necessárias para a implantação da norma, desenvolveu-se o escopo do projeto de implantação. Para tanto, construiu-se a EAP do projeto, a qual foi subdividida em quatro etapas: (i) iniciação; (ii) planejamento; (iii) execução; e (iv) encerramento, além de uma atividade 
constante de monitoramento e controle. Assim, a EAP foi dividida conforme o ciclo de vida do projeto de implantação da norma. A EAP do projeto é composta de 70 pacotes de trabalho, sendo 58 destes diretamente relacionados ao atendimento aos requisitos da ABNT NBR ISO/IEC 17025 e os outros 12 relacionados ao projeto e implantação.

Preliminarmente, a etapa iniciação é composta por apenas dois pacotes de trabalho e tem como seu foco principal a realização da reunião de abertura do projeto. Buscando alinhar as equipes do laboratório e do Escritório da Qualidade, é nesta reunião que ocorre o preenchimento do termo de abertura do projeto. Em seguida, a segunda etapa, planejamento, é composta por sete pacotes de trabalho, os quais envolvem a elaboração dos planos de gerenciamento do escopo, cronograma, aquisições, custos, recursos humanos, riscos e partes interessadas. Estes planos são elaborados em reuniões entre a equipe do projeto. A terceira etapa do projeto, execução, é quando ocorre de fato a implantação da norma nos laboratórios. Nesta etapa, realiza-se os 58 pacotes de trabalho identificados anteriormente.

Por fim, a quarta etapa do projeto, finalização, envolve três pacotes de trabalho. Inicialmente, realiza-se a primeira auditoria interna do laboratório, a qual deverá cobrir todos os elementos do SGQ recém implantado e deve seguir as diretrizes da ABNT NBR ISO 19011 (ABNT, 2019) e do procedimento de auditorias internas do SGLab CT. Também são realizados nesta última etapa do projeto, a elaboração do relatório final e a reunião de encerramento. Buscando a gestão do conhecimento desenvolvido ao longo do projeto, o relatório final deve conter um breve resumo de do andamento de todas as atividades do projeto, incluído as atividades de monitoramento e controle. Já a reunião de encerramento, têm como intuito a apresentação dos resultados do projeto às partes interessadas e deve ocorrer durante a reunião de análise crítica pela gerência do SGLab CT.

\subsection{Identificação das relações de dependência}

Com a Estrutura Analítica do Projeto (EAP) montada, estabeleceu-se as relações de dependência entre os pacotes de trabalho do projeto. Identificadas por meio de reuniões com a equipe coordenadora da implantação do SGQ nos laboratórios do SGLab CT, as relações de dependências foram classificadas em três categorias: Terminar para Começar (TC); Terminar para Terminar (TT); e Começar para Começar (CC), onde o tipo de relação de dependência mais comum no projeto é Terminar para Começar (TC). Embora relações do tipo Começar para Terminar (CT) também sejam possíveis teoricamente, não foram visualizadas dependências desse tipo neste estudo. As relações de dependência entre os pacotes de trabalho podem ser visualizadas na Tabela 2. 
Tabela 2

Relações de dependência do projeto de implantação do SGQ do SGLab CT

\begin{tabular}{|c|c|c|}
\hline EAP & Pacote de trabalho & Dependência \\
\hline 1.1 & Termo de abertura do projeto & N/A \\
\hline 1.2 & Reunião de abertura & $1.1(\mathrm{TC})$ \\
\hline 2.1 & Planejamento do escopo & $1(\mathrm{TC})$ \\
\hline 2.2 & Planejamento do cronograma & $2.1(\mathrm{TC})$ \\
\hline 2.3 & Planejamento de aquisições & $2.1(\mathrm{TC})$ \\
\hline 2.4 & Planejamento de custos & $2.3(\mathrm{TC})$ \\
\hline 2.5 & Planejamento de recursos humanos & 2.2. (TC) \\
\hline 2.6 & Planejamento do gerenciamento de riscos & 2.4 (TC); 2.5 (TC) \\
\hline 2.7 & Planejamento do gerenciamento de partes interessadas & $2.6(\mathrm{TC})$ \\
\hline 3.1.1 & Estabelecer laboratório & $2(\mathrm{TC})$ \\
\hline 3.1.2 & Estrutura organizacional & $2(\mathrm{TC})$ \\
\hline 3.1 .3 & Seleção de pessoal (Inicial) & $3.1 .2(\mathrm{TC})$ \\
\hline 3.1.4 & Gerência & $3.1 .2(\mathrm{TC}) ; 3.1 .3(\mathrm{CC})$ \\
\hline 3.1.5 & Escopo de acreditação & $2(\mathrm{TC})$ \\
\hline 3.1 .6 & Aquisição de equipamentos & $3.1 .5(\mathrm{TC})$ \\
\hline 3.1.7 & Instalações físicas & 3.1 .5 (TC); $3.1 .6(\mathrm{TT})$ \\
\hline 3.1.8 & Diretrizes do sistema & $2(\mathrm{TC})$ \\
\hline 3.2 .1 & Sistemática de documentos & $3.1 .8(\mathrm{TC})$ \\
\hline 3.2.2 & Sistemática de registros & $3.2 .1(\mathrm{TC})$ \\
\hline 3.3.1 & Procedimento(s) de pessoal & $3.2(\mathrm{TC})$ \\
\hline 3.3.2 & $\begin{array}{l}\text { Requisitos de competência, responsabilidades, autoridades e } \\
\text { inter-relacionamento }\end{array}$ & $3.3 .1(\mathrm{TC})$ \\
\hline 3.3.3 & Registros de pessoal & $3.3 .2(\mathrm{CC})$ \\
\hline 3.3.4 & Autorização de pessoal & $3.3 .3(\mathrm{TC})$ \\
\hline 3.4.1 & Requisitos para instalações e condições ambientais & 3.1.7 (TT); 3.3.4 (TC) \\
\hline 3.4 .2 & Medidas de controle das instalações & $3.4 .1(\mathrm{TC})$ \\
\hline 3.4.3 & Monitoramento, controle e registro das condições ambientais & $3.4 .1(\mathrm{TC})$ \\
\hline 3.5 .1 & Procedimento de equipamentos & $3.1 .6(\mathrm{TT}) ; 3.3 .4(\mathrm{TC})$ \\
\hline 3.5 .2 & Identificação de equipamentos & $3.5 .1(\mathrm{TC})$ \\
\hline 3.5.3 & Registros de equipamentos & $3.5 .1(\mathrm{CC})$ \\
\hline 3.5.4 & Programa de calibração & 3.5.1 (TC) \\
\hline 3.5 .5 & Procedimento para checagens intermediárias & $3.5 .1(\mathrm{TC})$ \\
\hline 3.6 & Rastreabilidade metrológica & 3.3.4 (TC); 3.5 .4 (TT) \\
\hline 3.7.1 & Procedimento de produtos e serviços providos externamente & 3.3.4 (TC) \\
\hline 3.7 .2 & Registros de produtos e serviços providos externamente & 3.7.1 (CC) \\
\hline 3.7 .3 & Seleção de provedores externos & $3.7 .1(\mathrm{TC})$ \\
\hline 3.8 .1 & Seleção de métodos & $\begin{array}{l}3.1 .5(\mathrm{TC}) ; 3.1 .6(\mathrm{TC}) \text {; } \\
3.1 .7(\mathrm{TC}) ; 3.3 .4(\mathrm{TC})\end{array}$ \\
\hline 3.8.2 & Sistemática de verificação/validação de métodos & $3.8 .1(\mathrm{TC})$ \\
\hline 3.8.3 & Verificação e/ou validação de métodos & $3.8 .2(\mathrm{TC})$ \\
\hline
\end{tabular}




\begin{tabular}{|c|c|c|}
\hline EAP & Pacote de trabalho & Dependência \\
\hline 3.8 .4 & Documentação dos métodos selecionados & $\begin{array}{l}3.8 .3(\mathrm{TC}) ; 3.9 .1(\mathrm{TT}) \\
3.18 .3(\mathrm{TT})\end{array}$ \\
\hline 3.9 .1 & Plano e método de amostragem & $3.8 .1(\mathrm{TC})$ \\
\hline 3.9 .2 & Registros de amostragem & $3.9 .1(\mathrm{CC})$ \\
\hline 3.10 & Incerteza de medição & $3.8 .4(\mathrm{TC})$ \\
\hline 3.11 .1 & $\begin{array}{l}\text { Procedimento de análise crítica de pedidos, propostas e } \\
\text { contratos }\end{array}$ & $3.3 .4(\mathrm{TC})$ \\
\hline 3.11 .2 & Registros de análises críticas & $3.11 .1(\mathrm{CC})$ \\
\hline 3.12 .1 & Procedimento para itens de ensaio/calibração & $3.8 .4(\mathrm{TT})$ \\
\hline 3.13 & Registros técnicos & $3.2 .2(\mathrm{CC}) ; 3.8 .4(\mathrm{CC})$ \\
\hline 3.14 .1 & Procedimento para monitorar a validade dos resultados & $3.8 .4(\mathrm{TT})$ \\
\hline 3.14 .2 & Monitoramento do desempenho & $3.14 .1(\mathrm{TC})$ \\
\hline 3.15 .1 & Sistemática para relato de resultados & $3.13 .1(\mathrm{TC})$ \\
\hline 3.15 .2 & Relatório de ensaio & $3.15 .1(\mathrm{TC})$ \\
\hline 3.15 .3 & Certificado de calibração & $3.15 .1(\mathrm{TC})$ \\
\hline 3.16 .1 & Procedimento de reclamações & $3.3 .4(\mathrm{TC})$ \\
\hline 3.17 .1 & Procedimento de tratamento de trabalho não conforme & $3.8 .4(\mathrm{TC})$ \\
\hline 3.17 .2 & Registros de trabalho não conforme & $3.17 .1(\mathrm{CC})$ \\
\hline 3.18 .1 & Sistemática de gestão da informação laboratorial & $3.1 .5(\mathrm{TC})$ \\
\hline 3.18 .2 & Registros de sistema de gestão da informação & $3.18 .1(\mathrm{TC})$ \\
\hline 3.18 .3 & $\begin{array}{l}\text { Validação do sistema de gestão da informação e controles de } \\
\text { dados }\end{array}$ & $3.18 .1(\mathrm{TC}) ; 3.18 .2(\mathrm{TC})$ \\
\hline 3.19 .1 & $\begin{array}{l}\text { Identificação de riscos e oportunidades associados as } \\
\text { atividades de laboratório }\end{array}$ & $3.8 .4(\mathrm{TT})$ \\
\hline 3.19 .2 & Ações para abordar riscos e oportunidades & $3.19 .1(\mathrm{TC})$ \\
\hline 3.20 & Melhoria & $3.3 .4(\mathrm{TC})$ \\
\hline 3.21 .1 & Sistemática para tratamento de não conformidades & $3.3 .4(\mathrm{TC})$ \\
\hline 3.21 .2 & Registros de ações corretivas & $3.21 .1(\mathrm{TT})$ \\
\hline 3.22 .1 & Intervalo de auditorias internas & $3.3 .4(\mathrm{TC})$ \\
\hline 3.22 .2 & Programa de auditoria interna & $3.22 .1(\mathrm{TC})$ \\
\hline 3.23 .1 & Intervalo de análise crítica pela gerência & $3.22(\mathrm{TC})$ \\
\hline 3.23 .2 & Registro de entradas para análise crítica pela gerência & $3.23 .1(\mathrm{TC})$ \\
\hline 3.23 .3 & Registro de saídas da análise crítica pela gerência & $3.23 .1(\mathrm{TC})$ \\
\hline 4.1 & Auditoria interna & $3(\mathrm{TC})$ \\
\hline 4.2 & Relatório final & $4.1(\mathrm{TC})$ \\
\hline 4.3 & Reunião de encerramento & $4.2(\mathrm{TC})$ \\
\hline
\end{tabular}

Fonte: Elaborada pelos autores.

Com base no escopo definido e nas relações de dependências visualizadas na Tabela 2, o cronograma do projeto de implantação de um SGQ baseado na ABNT NBR ISO/IEC 17025 pode ser construído para cada laboratório que receberá a implantação. Ressalta-se que a duração estimada de cada pacote de trabalho dependerá dos recursos disponíveis e da situação em cada contexto de 
implantação, porém a estruturação do escopo e das relações de dependência resultantes desse trabalho devem permanecer inalteradas e servir como base para cada projeto de implantação.

\subsection{Práticas e ferramentas de GP aplicáveis à implantação de um SGQ baseado na ABNT NBR ISO/IEC 17025}

Uma vez definidos a abordagem de GP, os pacotes de trabalho, a EAP e as relações de dependência, práticas e ferramentas de GP foram identificadas para auxiliar na condução da implantação do SGQ baseado na ABNT NBR ISO/IEC 17025 através de um projeto. Para a fase de iniciação, optou-se pela utilização do Project Model Canvas (PM Canvas) como modelo para o termo de abertura do projeto. Segundo Finocchio Júnior (2013), o PM Canvas busca desenvolver um plano de projeto de maneira efetiva e amigável que, quando utilizado preliminarmente, servirá como base para as diretrizes do projeto. O PM Canvas foi adaptado à realidade do SGLab CT, conforme apresentado na Figura 2.

\section{Figura 2}

PM Canvas adaptado

GP:

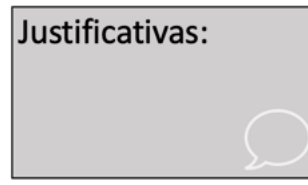

Objetivo SMART:

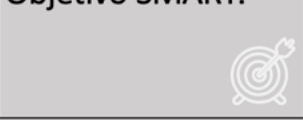

Benefícios:
PITCH:
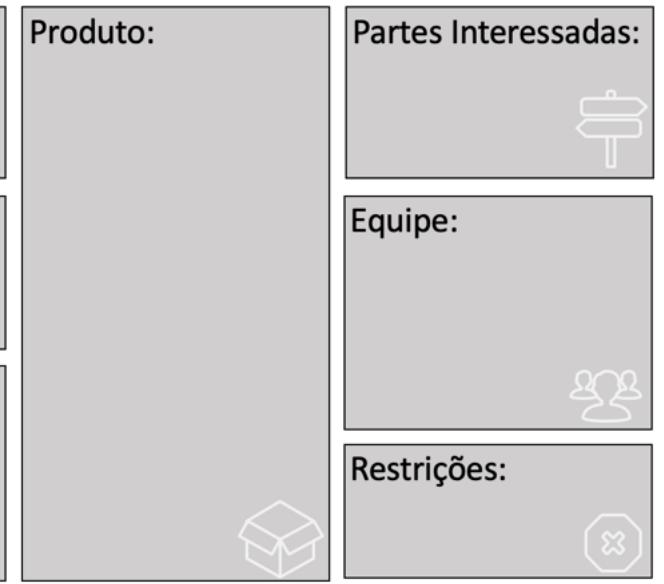

Equipe:

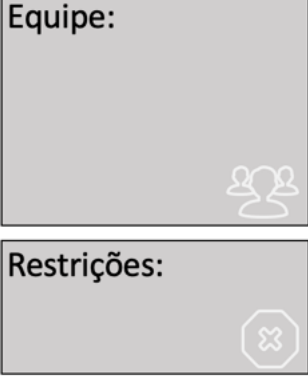

Fonte: Adaptado de Finocchio Júnior (2013).

Como a metodologia dominante do projeto seria baseada na abordagem tradicional trazida pelo Guia PMBOK ${ }^{\circ}$, estabeleceu-se que para as fases de planejamento e execução seria vantajosa a utilização de um software para facilitar o gerenciamento do projeto. Quatro opções de softwares foram analisadas: Microsoft Project ; Bitrix24; Gantt Excel; e, TeamGantt. Após um comparativo de benefícios e limitações, selecionou-se o TeamGantt, uma vez que esse possui um plano gratuito, pode ser acessado on-line, possui interface de fácil aprendizado, e possui recursos de controles do escopo de recursos. 
Embora o TeamGantt ofereça uma série de recursos úteis para o estabelecimento do cronograma do projeto (baseados na EAP e nas relações de dependências previamente estabelecidas), o software não dispõe de funções para o controle de riscos, aquisições, custos e o monitoramento de partes interessadas. Assim, para o monitoramento e controle do projeto, elaborou-se uma planilha de acompanhamento no software Excel ${ }^{\circ}$, onde é possível o lançamento das aquisições planejadas, não planejadas e o seus respectivos status; os custos planejados, não planejados, bem como o desvio entre o real e o estimado; os riscos do projeto e as ações de mitigação; e o monitoramento do engajamento das partes interessadas no projeto. Ressalta-se que, nesta pesquisa, o controle de recursos se limitou aos recursos humanos, uma vez que a universidade na qual os laboratórios estão inseridos já dispõe de políticas para o controle de outros tipos de recursos.

Outra prática de GP identificada para a execução e monitoramento do projeto foi a realização de reuniões de acompanhamento, as quais foram estruturadas com base no Scrum, em linha com a abordagem híbrida definida. Tais reuniões foram divididas em duas categorias: reuniões diárias e reuniões de trabalho. A primeira, deve ser realizada conforme as reuniões diárias do Scrum e devem ocorrer separadamente entre cada área do SGLab CT, laboratório e Escritório da Qualidade. Em contrapartida, as reuniões de trabalho são integradas, ocorrem semanalmente e estão subdivididas em três seções: status das atividades; treinamentos; e, realização e verificação de tarefas. Ao final de cada bimestre, durante a última reunião de trabalho, deve-se analisar os resultados alcançados no período e avaliar o andamento das atividades, buscando identificar oportunidades de melhoria para o próximo período. Esta prática é inspirada nos Sprints da metodologia Scrum.

\section{Discussão}

A implantação de um SGQ baseado na ISO/IEC 17025 é uma tarefa complexa, dado o elevado número de requisitos a serem atendidos. Nesse sentido, a utilização da abordagem de GP demonstrada nessa pesquisa pode trazer benefícios, facilitando a consolidação do SGQ para o laboratório ou conjunto de ensaios ou calibrações em que se deseja realizar a implantação. Embora na literatura a abordagem de processos seja mais comum, um comparativo entre a abordagem de processos e de GP pode ser realizada. Por exemplo, a Tabela 3 apresenta um comparativo entre as nove etapas sugeridas por Grochau e ten Caten (2012) para implantação de um SGQ baseado na ISO/IEC 17025 por meio da abordagem de processos e os resultados dessa pesquisa, onde pode-se notar que os objetivos pretendidos com cada etapa na abordagem de Grochau e ten Caten (2012) também são satisfeitos pela abordagem de GP. 
Tabela 3

Comparativo entre a abordagem de processos de Grochau e ten Caten (2012) e os resultados da pesquisa

\begin{tabular}{l|l}
$\begin{array}{l}\text { Etapas para a implantação de } \\
\text { um SGQ (Grochau e ten Caten, } \\
\text { 2012) }\end{array}$ & $\begin{array}{c}\text { Objetivos da etapa (Grochau e ten } \\
\text { Caten, 2012) }\end{array}$ \\
$\begin{array}{ll}\text { (i) Pré-definir equipe e escopo } \\
\text { da acreditação }\end{array}$ & $\begin{array}{l}\text { Identificar em quais laboratórios } \\
\text { ou ensaios se fará a implantação } \\
\text { do SGQ, bem como as pessoas } \\
\text { envolvidas e suas } \\
\text { responsabilidades. }\end{array}$ \\
\hline
\end{tabular}

(ii) Realizar diagnóstico da situação atual

Identificar as necessidades e os recursos demandados para cumprir com os requisitos da ISSO/IEC 17025.

Orçar os custos necessários para a

(iii) Calcular os custos da implantação implantação considerando as necessidades verificadas na etapa (ii).

Definir prazos e responsáveis para

as atividades.

(iv) Elaborar cronograma definir responsabilidades

(v) Mapear e analisar processos

(vi) Estabelecer os requisitos de gerenciamento do SGQ

(vii) Estabelecer os requisitos técnicos do SGQ

(viii) Definir e acompanhar indicadores

Avaliar o nível de atendimento dos objetivos propostos
Como os objetivos de cada etapa são cumpridos na abordagem de GP?

O escopo da acreditação e a equipe são definidos nos itens 1.1. e 1.2 da EAP, com auxílio do PM Canvas adaptado mostrado na Figura 2.

O cumprimento de toda a etapa 3 (execução) da EAP depende, inicialmente, do estabelecimento da situação atual para direcionar as necessidades do que precisa ser desenvolvimento em cada pacote de trabalho.

A estimativa dos custos do projeto de implantação está prevista no item 2.4 da EAP, sendo realizada por meio de um plano de gerenciamento de custos.

O cronograma é elaborado do item 2.2. da EAP, observando as relações de dependência apresentadas na Tabela 2 e com o uso recomendado da ferramenta TeamGantt.

O cumprimento dos requisitos da ISO/IEC 17025 é realizado por meio dos 58 pacotes de trabalho, apresentados na Tabela 1.

Os requisitos da ISO/IEC 17025, após análise do mapa mental, foram desdobrados em pacotes de trabalho para sua implantação, conforme Tabela 1.

O acompanhamento de indicadores de custos, riscos, e engajamento das partes interessadas é realizado com o uso de planilha eletrônica, enquanto a variação (previsto $x$ realizado) do cronograma pode ser feita diretamente no TeamGantt.

A avaliação do projeto de implantação do SGQ é realizada por meio de auditorias (interna e final), conforme itens 4.1 e 4.2 da EAP.

Fonte: Elaborada pelos autores.

Identificar os desvios e não conformidades do SGQ e planejar as ações necessárias. 
entanto, a análise realizada nessa pesquisa prevê o desdobrando dos requisitos da ISO/IEC 17025 em 58 pacotes de trabalho, trazendo, portanto, maior detalhamento e tornando mais clara a divisão do trabalho a ser realizado para a implantação do SGQ. Além destes, 12 pacotes de trabalho adicionais foram propostos na construção da EAP, de modo que o conjunto final de 70 pacotes de trabalho representa as atividades necessárias de todo o ciclo de vida do projeto de implantação do SGQ, desde a iniciação, planejamento, execução e encerramento.

A implantação de um SGQ pela abordagem de GP, no entanto, não invalida o uso da abordagem de processos no gerenciamento do SGQ. Este conflito pode ser mitigado pela distinção entre implantação do SGQ e operação e manutenção do SGQ. Considerando que a implantação é um esforço temporário para criar um resultado único, a mesma pode ser melhor gerenciada pela abordagem de GP, visto que atende a definição de projeto (PMI, 2018). A vantagem, nesse caso, é o uso de ferramentas tradicionalmente associadas ao conhecimento de GP e ao contexto de projetos, como a definição de pacotes de trabalho, EAP, relações de dependência, entre outros. Por outro lado, uma vez implantado o SGQ, a operação dos laboratórios e dos ensaios em conformidade com o SGQ torna-se parte da rotina da organização, de maneira continuada, e, portanto, atende as definições de processo (Baiyere et al., 2020). Dessa forma, a operação do SGQ pode ser melhor gerenciada com base nessa abordagem, que trata o funcionamento da organizações como uma rede interconectada de processos e dispõem de ferramentas adequadas para mapear e melhorar processos, como por exemplo a Business Process Model and Notation (BPMN) (Stravinskiene \& Serafinas, 2020).

Por fim, a abordagem de GP também traz vantagens específicas para o contexto de implantação de SGQs em IES. Dado que em laboratórios de IES é esperado que os envolvidos desempenhem múltiplas funções organizacionais e realizam atividades de diversas naturezas (Grochau et al., 2010; Rodima et al., 2005), o que pode ser uma barreira à implantação do SGQ (Lovrenčić Mikelić, 2020; Zapata-García et al., 2007), o desdobrando do projeto de implantação em 70 pacotes de trabalhos, devidamente sequenciados utilizando-se as relações lógicas de dependências (TC, TT, CC e TC) torna inequívoco o trabalho a ser feito e seu ordenamento. A utilização da abordagem de GP, portanto, pode ser útil para conciliar as atividades acadêmicas desenvolvidas nestes laboratórios com os esforços de implantação de $\mathrm{SGQ}$, visto que o cronograma desenvolvido pode prever o agendamento para as atividades de implantação considerando também as necessidades de uso desses espaços para o ensino, pesquisa e extensão.

\section{Conclusão}

Buscando-se aprimorar o processo de implantação de um SGQ baseado na ABNT NBR ISO/IEC 17025, o presente estudo teve como objetivo estruturar a implantação de um SGQ baseados na norma ABNT NBR ISO/IEC 17025 em laboratórios de ensaio e calibração de uma Instituição de Ensino Superior 
utilizando a abordagem de gestão de projetos. Para a realização desta pesquisa, inicialmente foi necessária a definição da metodologia de Gerenciamento de Projetos a ser aplicada, buscando identificar a abordagem e as ferramentas mais adequadas à realidade do SGLab CT. A partir desta análise, optou-se pela utilização de uma abordagem híbrida, com a abordagem tradicional como dominante. Após, elaborou-se o escopo do projeto de implantação do $S G Q$, onde realizou-se uma extensa análise de todos os requisitos da norma ABNT NBR ISO/IEC 17025. 70 pacotes de trabalho que formam a EAP foram estabelecidos, sendo 58 destes diretamente relacionados ao atendimento aos requisitos da norma e 12 relacionados ao projeto de implantação. Práticas e ferramentas de GP foram elencadas para suportar o processo de implantação do SGQ em cada laboratório.

Assim, pode-se afirmar que o objetivo da pesquisa foi atingido, trazendo como conclusão teórica a demonstração de que as metodologias de Gerenciamento de Projetos são aplicáveis ao processo de implantação da ABNT NBR ISO/IEC 17025 e podem facilitar a implantação da mesma, uma vez que deixam claro o escopo da implantação, o trabalho a ser feito e o ordenamento das atividades com base nas relações de dependência. Além disso, práticas e ferramentas de GP auxiliam na execução e monitoramento e controle do trabalho, aumentando-se as probabilidades de sucesso. Como conclusão prática, os resultados dessa pesquisa podem ser utilizados por gestores e decisores que estejam liderando a implantação de SGQs baseados na ISO/IEC 17025 ou em outras normas. Como conclusão para a sociedade, espera-se que os resultados dessa pesquisa auxiliem especialmente laboratórios de IES na implantação de SGQs baseados na ISO/IEC 17025 ou em outras normas, oferecendo assim resultados com qualidade e confiabilidade no ensino, pesquisa, extensão e prestação de serviços.

Como estudos futuros, sugere-se a avaliação da eficiência do uso de metodologias de GP por meio da comparação dos resultados dos projetos com implantações realizadas por meio de outros tipos de abordagens. Outra possibilidade de estudo é a comparação entre os resultados de projetos de implantação de normas com o uso de diferentes abordagens, práticas e ferramentas de GP.

\section{Referências}

ABNT. (2017). ABNT NBR ISO/IEC 17025:2017: Requisitos gerais para a competência de laboratórios de ensaio e calibração. Associação Brasileira de Normas Técnicas (ABNT).

ABNT. (2019). ABNT NBR ISO/IEC 19011:2018 Versão Corrigida:2019: Diretrizes para auditoria de sistemas de gestão. Associação Brasileira de Normas Técnicas (ABNT).

Albano, F. de M., \& Raya-Rodriguez, M. T. (2009). Validação e garantia da qualidade de ensaios laboratoriais. Rede Metrológica RS.

Aqidawati, E. F., Sutopo, W., \& Zakaria, R. (2019). Model to measure the readiness of university testing laboratories to fulfill ISO/IEC 17025 requirements (a case study). Journal of Open Innovation: Technology, Market, and Complexity, 5(1), 1-19. https://doi.org/10.3390/joitmc5010002 
Baiyere, A., Salmela, H., \& Tapanainen, T. (2020). Digital transformation and the new logics of business process management. European Journal of Information Systems, 29(3), 238-259. https://doi.org/10.1080/0960085X.2020.1718007

Barnes, T. A., Pashby, I. R., \& Gibbons, A. M. (2006). Managing collaborative R\&D projects development of a practical management tool. International Journal of Project Management, 24(5), 395-404. https://doi.org/10.1016/j.ijproman.2006.03.003

Chung, K. H., Choi, G. S., Lee, W., Cho, Y. H., \& Lee, C. W. (2006). Implementation of ISO/IEC 17025 standard in a nuclear analytical laboratory: The KAERI experience. Accreditation and Quality Assurance, 10(11), 603-605. https://doi.org/10.1007/s00769-005-0060-1

Cruz, F. (2013). Scrum e PMBOK unidos no gerenciamento de projetos. Brasport.

Eder, S., Conforto, E. C., Amaral, D. C., \& Silva, S. L. da. (2014). Diferenciando as abordagens tradicional e ágil de gerenciamento de projetos. Production, 25(3), 482-497.

https://doi.org/10.1590/S0103-65132014005000021

Eder, S., Conforto, E. C., Schnetzler, J. P., Amaral, D. C., \& Silva, S. L. da. (2012). Estudo das práticas de gerenciamento de projetos voltadas para desenvolvimento de produtos inovadores. Produto \& Produção, 13(1). https://doi.org/10.22456/1983-8026.24600

Felippes, B. A. de, Aguiar, J. G., \& Diniz, A. C. G. C. (2011). Sistema da qualidade em laboratórios universitários: incentivo ao ensino, pesquisa e extensão. Revista de Ensino de Engenharia, 30(2), 14-23. http://revista.educacao.ws/revista/index.php/abenge/article/view/99/79

Fernandes, G., Pinto, E. B., Araújo, M., \& Machado, R. J. (2020). The roles of a Programme and Project Management Office to support collaborative university-industry R\&D. Total Quality Management and Business Excellence, 31(5-6), 583-608. https://doi.org/10.1080/14783363.2018.1436963

Finocchio Júnior, J. (2013). Project Model Canvas - Gerenciamento de Projetos Sem Burocracia. Elsevier-Campus.

Grochau, I. H., Caten, C. S. ten, \& Forte, M. M. de C. (2015). Cenário Brasileiro na acreditação de laboratórios. Anais Do Congresso Brasileiro de Metrologia, 1-5.

http://bom.org.br:8080/xmlui/handle/2050011876/878

Grochau, I. H., Ferreira, C. A., Ferreira, J. Z., \& ten Caten, C. S. (2010). Implementation of a quality management system in university test laboratories: a brief review and new proposals. Accreditation and Quality Assurance, 15(12), 681-689. https://doi.org/10.1007/s00769-0100713-6

Grochau, I. H., \& ten Caten, C. S. (2012). A process approach to ISO/IEC 17025 in the implementation of a quality management system in testing laboratories. Accreditation and Quality Assurance, 17(5), 519-527. https://doi.org/10.1007/s00769-012-0905-3

Highsmith, J. (2009). Agile Project Management: Creating Innovative Products. Addison-Wesley Professional.

Ingason, H. T. (2015). Best Project Management Practices in the Implementation of an ISO 9001 Quality Management System. Procedia - Social and Behavioral Sciences, 194(October 2014), 192-200. https://doi.org/10.1016/j.sbspro.2015.06.133 
INMETRO. (2018). DOQ-CGCRE-087: Orientações gerais sobre os requisitos da ABNT NBR ISO/IEC 17025:2017. Instituto Nacional de Metrologia, Qualidade e Tecnologia (INMETRO).

Jackson, M. B. (2012). Step by step. PM Network, 26(6), 56-61.

https://www.pmi.org/learning/library/step-iterative-waterfall-approach-4303

Kerzner, H. R. (2015). Gerenciamento de projetos: uma abordagem para planejamento, programação e controle. Blucher.

Lopes, I., Santos, L., Pereira, M. F., Vaz, P., \& Alves, J. G. (2014). Implementation of the quality management system at the Laboratory of Radiological Protection and Safety (LPSR) in Portugal. Accreditation and Quality Assurance, 19(5), 355-360. https://doi.org/10.1007/s00769-014-1073-4

Lovrenčić Mikelić, I. (2020). Accreditation in Croatia: What is the position of testing and calibration laboratories from the science and higher education system? Accreditation and Quality Assurance, 25(3), 243-252. https://doi.org/10.1007/s00769-020-01430-y

Menezes, L. C. de M. (2018). Gestão de Projetos. Atlas.

Netto, D. A. M., Mendes, M. D. L., Coelho, R. R., Ribeiro, P. E. de A., \& Marion, M. L. P. (2009). Acreditação de ensaios do Laboratório de Análise de Sementes da Embrapa Milho e Sorgo pela ISO/IEC 17025:2005 e obtenção do Renasem. https://www.infoteca.cnptia.embrapa.br/bitstream/doc/658911/1/Com167.pdf

PMI. (2018). Um Guia de Conhecimento em Gerenciamento de Projetos (GUIA PMBOK) (6th ed.). Project Management Institute Inc.

Rauret, G., \& Compañó, R. (2003). Quality Management in Undergraduate Laboratories. A Systematic Approach to its Implementation. Microchimica Acta, 142(3), 177-185. https://doi.org/10.1007/s00604-003-0009-1

Rodima, A., Vilbaste, M., Saks, O., Jakobson, E., Koort, E., Pihl, V., Sooväli, L., Jalukse, L., Traks, J., Virro, K., Annuk, H., Aruoja, K., Floren, A., Indermitte, E., Jürgenson, M., Kaleva, P., Kepler, K., \& Leito, I. (2005). ISO 17025 quality system in a university environment. Accreditation and Quality Assurance, 10(7), 369-372. https://doi.org/10.1007/s00769-005-0011-x

Salgueiro, C. R. (2012). O desafio da implantação de um sistema de gestão da qualidade em laboratórios de pesquisa que realizam ensaios ecotoxicológicos. Universidade Estadual de Campinas. https://doi.org/10.47749/T/UNICAMP.2012.896628

SGLab, S. de G. de L. (2020). Manual da Qualidade. Universidade Federal de Santa Maria. https://www.ufsm.br/app/uploads/sites/809/2020/05/MQ-SGLab-CT-R08.pdf

Stravinskiene, I., \& Serafinas, D. (2020). The Link between Business Process Management and Quality Management. Journal of Risk and Financial Management, 13(225), 1-11. https://doi.org/10.3390/jrfm13100225

Vargas, R. V. (2018). Gerenciamento de Projetos: Estabelecendo Diferenciais Competitivos (9th ed.). Brasport.

Zapata-García, D., Llauradó, M., \& Rauret, G. (2007). Experience of implementing ISO 17025 for the accreditation of a university testing laboratory. Accreditation and Quality Assurance, 12(6), 317-322. https://doi.org/10.1007/s00769-007-0274-5 
Apêndice A

EAP do projeto de implantação do SGQ do SGLAB CT

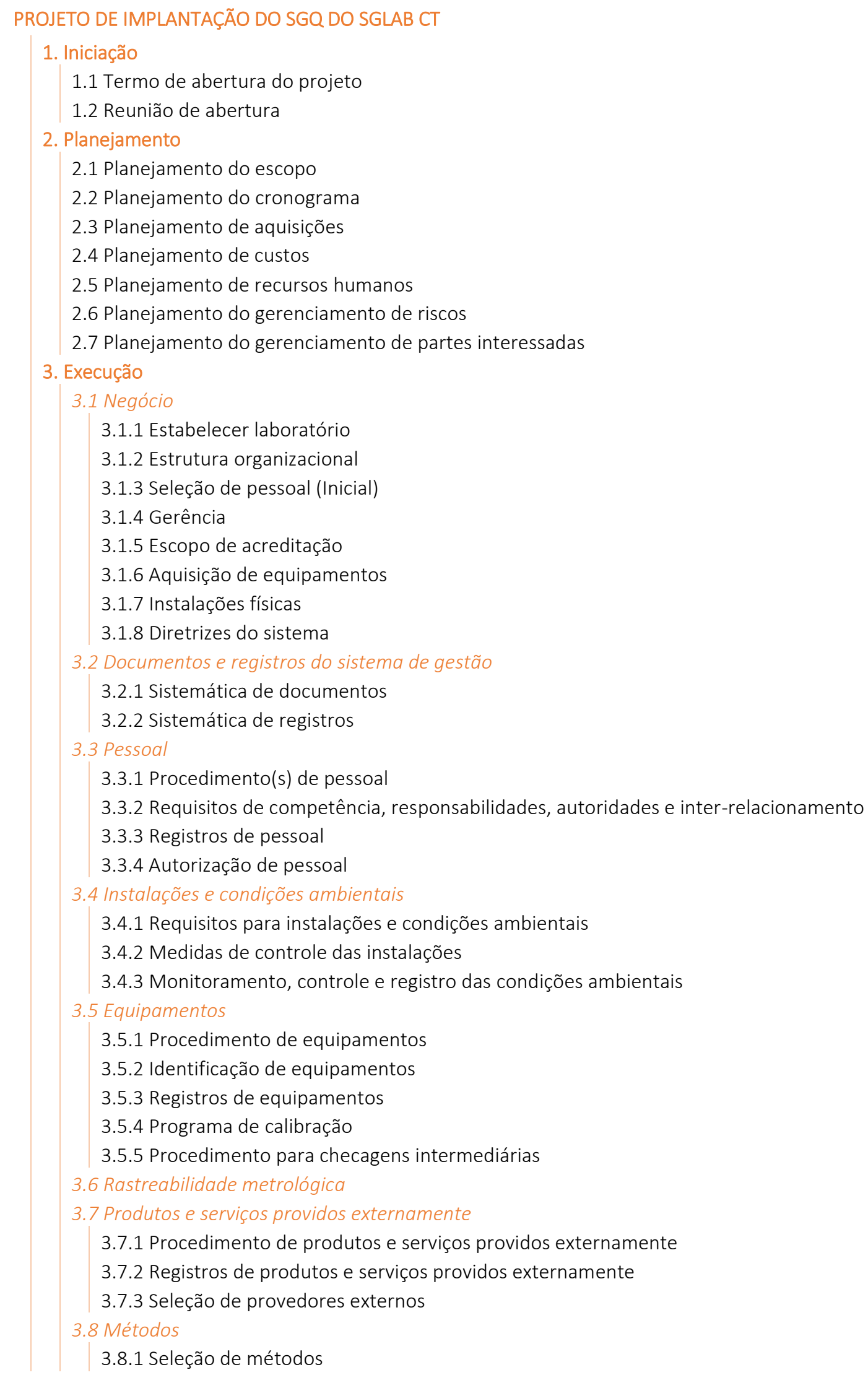


3.8.2 Sistemática de verificação/validação de métodos

3.8.3 Verificação e/ou validação de métodos

3.8.4 Documentação dos métodos selecionados

3.9 Amostragem

3.9.1 Plano e método de amostragem

3.9.2 Registros de amostragem

3.10 Incerteza de medição

3.11 Análise crítica de pedidos, propostas e contratos

3.11.1 Procedimento de análise crítica de pedidos, propostas e contratos

3.11.2 Registros de análises críticas

3.12 Manuseio de itens de ensaio ou calibração

3.12.1 Procedimento para itens de ensaio/calibração

3.13 Registros técnicos

3.14 Garantia da validade de resultados

3.14.1 Procedimento para monitorar a validade dos resultados

3.14.2 Monitoramento do desempenho

3.15 Relato de resultados

3.15.1 Sistemática para relato de resultados

3.15.2 Relatório de ensaio

3.15.3 Certificado de calibração

3.16 Reclamações

3.16.1 Procedimento de reclamações

3.17 Trabalho não conforme

3.17.1 Procedimento de tratamento de trabalho não conforme

3.17.2 Registros de trabalho não conforme

3.18 Controle de dados e gestão da informação

3.18.1 Sistemática de gestão da informação laboratorial

3.18.2 Registros de sistema de gestão da informação

3.18.3 Validação do sistema de gestão da informação e controles de dados

3.19 Riscos e oportunidades

3.19.1 Identificação de riscos e oportunidades associados as atividades de laboratório

3.19.2 Ações para abordar riscos e oportunidades

3.20 Melhoria

3.21 Ações corretivas

3.21.1 Sistemática para tratamento de não conformidades

3.21.2 Registros de ações corretivas

3.22 Auditorias internas

3.22.1 Intervalo de auditorias internas

3.22.2 Programa de auditoria interna

3.23 Análise crítica pela gerência

3.23.1 Intervalo de análise crítica pela gerência

3.23.2 Registro de entradas para análise crítica pela gerência

3.23.3 Registro de saídas da análise crítica pela gerência

4. Encerramento

4.1 Auditoria interna

4.2 Relatório final

4.3 Reunião de encerramento 